\title{
Hepatoprotective Effect of Green Tea Extract against Cyclophosphamide Induced Liver Injury in Albino Rats
}

\author{
Ali Hassan A. Ali ${ }^{1,2}$ \\ ${ }^{1}$ Anatomy Department, College of Medicine, Prince Sattam Bin Abdulaziz University, Al-Kharj, KSA \\ ${ }^{2}$ Anatomy Department, Faculty of Medicine, Al-Azhar University, Cairo, Egypt \\ Email: alihassan3750@yahoo.com
}

How to cite this paper: Ali, A.H.A. (2018) Hepatoprotective Effect of Green Tea Extract against Cyclophosphamide Induced Liver Injury in Albino Rats. Forensic Medicine and Anatomy Research, 6, 11-19. https://doi.org/10.4236/fmar.2018.62002

Received: March 9, 2018

Accepted: April 10, 2018

Published: April 13, 2018

Copyright $\odot 2018$ by author and Scientific Research Publishing Inc. This work is licensed under the Creative Commons Attribution International License (CC BY 4.0).

http://creativecommons.org/licenses/by/4.0/

\section{(c) (i) Open Access}

\begin{abstract}
Background: Green tea intake is accompanied with a lower incidence of cardiovascular disease, cancer and neurodegenerative disorders; hence green tea extract has been included as dietary supplement along with other supplements and multivitamins. Aim of the Work: Studying the effect of cyclophosphamide administration on the liver of adult male albino rats and the possible protective role of green tea extract. Material and Methods: The current study was carried out on 45 adult male albino rats. They were divided into three equal groups (each included 15 rats). Group I (control group) was injected intraperitoneally with normal saline at a dosage of $0.5 \mathrm{mg} / \mathrm{kg}$ body weight twice weekly for 9 weeks. Group II was injected intraperitoneally with cyclophosphamide (CP) $(150 \mathrm{mg} / \mathrm{kg} /$ day $)$ for two weeks. Group III: rats received green tea extracts orally $(50 \mathrm{mg} / \mathrm{kg} /$ day $)$ for three weeks, and then continued for further two weeks concomitantly with intraperitoneally cyclophosphamide (CP) injected $(150 \mathrm{mg} / \mathrm{kg} /$ day). Results: Rats exposed to cyclophosphamide (CP) showed several histological and histochemical changes in their liver. These changes were improved by using green tea. Conclusion: The present work showed that green tea had preventive and therapeutic effect upon livers of albino rats after they were exposed to CP.
\end{abstract}

\section{Keywords}

Green Tea, Albino Rats, Liver, Cyclophosphamide

\section{Introduction}

Nutritional antioxidants, in general, are often safe substances found in the medicinalplants and possess attractive effects in the complementary medicine including 
an important action in reduction of the oxidative stress [1] [2]. Polyphenols and catechins constituting significant nutritional antioxidants groups are particularly found in the green tea [3] [4]. In addition to their antioxidant action, these nutrient substances exhibit anti-diabetic, antihypertensive, anti-atherothrombogenic, anti-carcinogenic, anti-oxidative, anti-inflammatory, and anti-fungal functions [5] [6]. Therefore, consumption of green tea is accompanied with a lower mortality rate particularly from the cardiovascular strokes [7]. The mechanism of protection exerted by green tea consumption against chronic diseases is unobvious, however, it had been suggested that the protective role of green tea intake with these diseases might be resorted to the antioxidant effect of its polyphenols and catechins [4] [8] [9].

$\mathrm{CP}$ is a cytotoxic alkylating agent that is used extensively as antineoplastic agent for the treatment of various cancers, as well as an immunosuppressive agent for organ transplantation, multiple sclerosis, systemic lupus erythematosus and other benign tumors [10]. CP treatment is associated with oligospermia and azoospermia, as well as biochemical and histological alterations in the testis and epididymis of human and rats [11] [12] [13].

This study aimed to investigate the histological changes in the liver tissue of male albino rats which exposed to $\mathrm{CP}$ and the possible protective role of green tea.

\section{Material and Methods}

\subsection{Experimental Animals}

The current study was accomplished on forty-five adult male albino rats weighing 150 - $170 \mathrm{gm}$. They were obtained from the animal house of Prince Sattam Bin Abdulaziz University, the rats were housed in temperature controlled rooms $\left(25^{\circ} \mathrm{C}\right)$ with constant humidity $(40 \%-70 \%)$ and $12 \mathrm{~h}$ light/dark cycle prior to use in experimental protocols. Rats were fed a standard pellet diet and allowed free access to water throughout the study.

Green tea extract: was obtained as $300 \mathrm{mg}$ tablets synthesized by Tecno Med Company-KSA. The tablets were crushed and the required amount was dissolved in distilled water.

Experimental design: The 45 rats were housed in normal standard conditions; including cages, 55\% relative humidity, and central ventilation environment with $20^{\circ} \mathrm{C} \pm 2{ }^{\circ} \mathrm{C}$ temperature. Two weeks after accommodation phase, the rats were evenly divided into 3 groups (each included 15 rats). The three groups, that were kept on standard chow diet throughout the study.

The experimental animals were divided into 3 groups as following.

Group I (Control group): whose animals were intraperitoneally (IP) injected with $0.5 \mathrm{mg} / \mathrm{kg}$ body weight (BW) normal saline twice weekly for the total period of 9 weeks.

Group II (CP): rats were intraperitoneally injected with CP (150 mg/kg/day) for two weeks divided in two daily doses.

Group III (Green tea + CP): rats received green tea extract $(50 \mathrm{mg} / \mathrm{kg})$ orally 
via gastric tube once daily for three weeks before $\mathrm{CP}$ administration and continued for two weeks' concomitant with CP $(150 \mathrm{mg} / \mathrm{kg})$.

After sacrificing animals, their livers were processed, stained, and examined microscopically.

\subsection{Histopathological Technique}

All the three groups of rats were sacrificed at the end of our experiment by halothane overdose. Autopsy liver specimens were excised, washed several times with normal saline, and each specimen was instantly fixed with $10 \%$ neutral buffered formalin, washed in tap water, dehydrated in successive ethyl alcohol grades of concentrations, cleared in xylol, and embedded in paraffin to make tissue blocks. Then, 5 micron sections were cut, placed on charged glass slides, and stained with hematoxylin and eosin stain (Hx. \& Eo.) to examine structural morphology and detect histopathological changes, also stained with Mallory's trichrome stain to determine collagen fibers, in addition to staining with Periodic Acid-Schiff Reagent (PAS) method to identify the amount of cytoplasmic polysaccharides [14].

\subsection{Statistical Analysis}

Statistical analysis was done using the SPSS statistical version 22. All data were expressed as mean \pm standard deviation. P-values less than 0.05 were considered statistically significant. The results of both groups II and III were compared to those of group I (control group) and the results of group II as well were compared to those of group III by using the Paired-Samples " $t$ " test.

\section{Results}

\section{Histological and Histochemical Results}

In Group I, (Control, untreated negative control), investigations of normalliver sections, stained with Hematoxylin and Eosin (H\&E) revealed that each hepatic lobule consists of a central vein lined with simple squamous epithelium surrounded by polygonal hepatic cells radially distributed in the form of strands containing quite clear blood sinusoids in between (Figure 1(A)). Also, there is no lobular inflammation. The central vein did not contain lymphocytes and none of necrosis of liver cells. The hepatocytes are normally uni- or binucleated. The hepatocytic cytoplasm is eosinophilic and appeared homogenous with many uniformly distributed coarse basophilic granules. Hepatocytes nuclei are large, vesicular and contain one or more nucleoli. There are many spindle-shaped Kupffer's cells appeared between the hepatocytes with deeply-stained nuclei (Figure 1(A)). The hepatic portal tracts have branches of the hepatic portal vein, hepatic artery and the bile duct (Figure 2(B)). In CP group, liver sections of rats showed dilated hepatic sinusoids. Affected hepatocytes lost their nuclei with vacuolated cytoplasm and some other shad pyknotic nuclei (Figure 1(B)). Dilated hepatic portal veins filled with amyloidal substances as well as erythrocytes were 


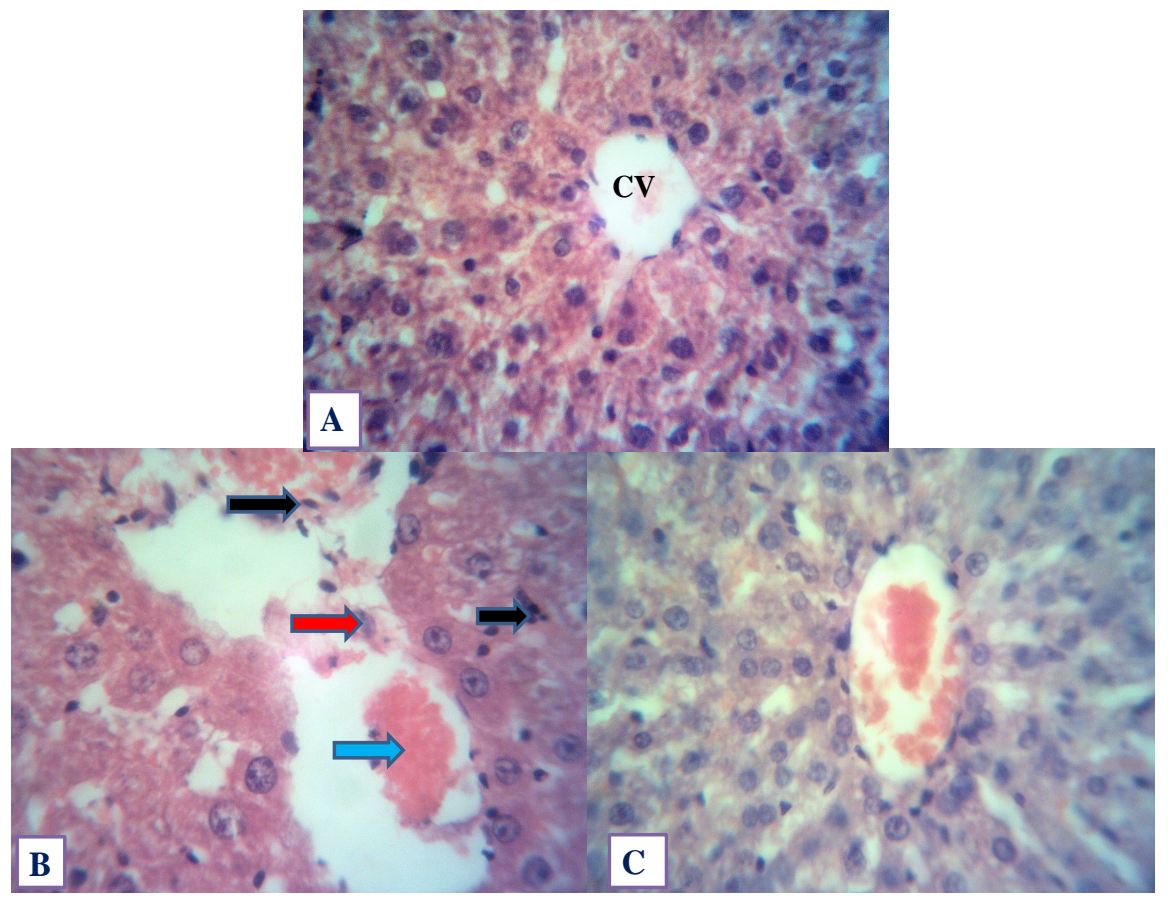

Figure 1. (A) A photomicrograph of the control liver of adult albino rat showing normal lobular pattern with a centrilobular vein $(\mathrm{CV})$ and radiating irregular branching and anastomosing plates of hepatocytes with intervening sinusoids lined with endothelial cells. Most of the hepatocytes have vesicular nuclei. (B) A photomicrograph of the liver of group II (CP) adult albino rat showing dilatation of central vein, hepatocytes necrotic changes such as marked hepatocytic ballooning, small pyknotic nuclei and fatty degeneration (black arrows) around the central vein. Congestion of central vein with hemolized blood (blue arrow) and little amount of cellular infiltration (red arrow) were detected. (C) A photomicrograph of group III (Green tea $+\mathrm{CP}$ ) showing that most of hepatic cellular structure and hepatic sinusoids are almost similar to that of the control group. (Hx. \& E. (A), (B) \& (C) $\times 400)$.

seen and its walls were thickened and invaded with many fibroblasts. Many pyknotic nuclei were also noticed surrounding the portal vein (Figure 2(B)). Also, progressive wideness of hepatic sinusoids as well as irregular central veins were detected. Furthermore, there was a remarkable proliferation in the cellular wall of a ruptured bile duct (Figure 2(B)). Hepatocellular necrosis appeared with lobular inflammation and lymphocytic infiltration. In Group III (Green tea + CP) considerable degree of improvement on the level of hepatic cellular structure, hepatic sinusoids and portal tracts was detected (Figure 1(C) \& Figure 2(C)). In Group 1, investigations of normal liver sections stained with Mallory's trichrome stain showed the appearance of little amounts of collagenous fibres around the hepatocytes and hepatic sinusoids in the form of fine threads of collagen fibres around central vein (Figure 3(A)). Also, fine threads of collagen fibres were detected in the portal area surround the hepatic portal vein and bile duct (Figure 4(A)). In Group II, liver sections of rats after taking CP showed an obvious significant increase in the collagenous fibres deposition around hepatic sinusoids, central vein and portal tract structures in comparison to the control 


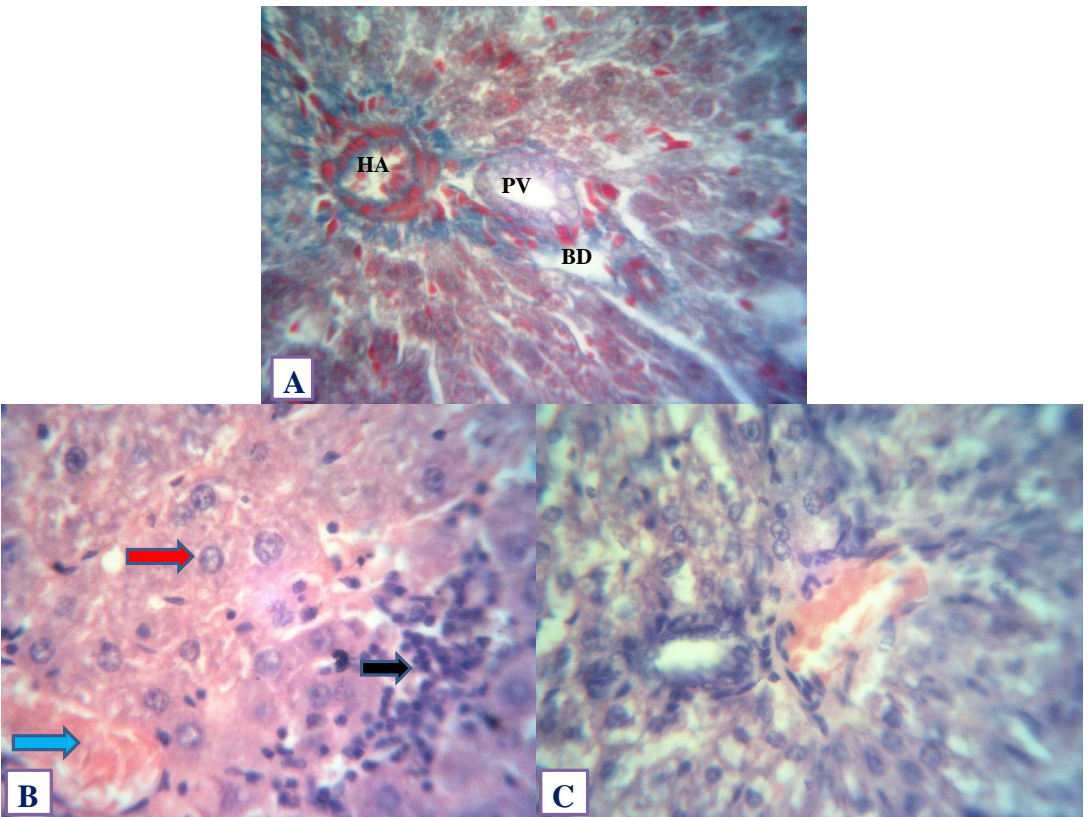

Figure 2. (A) A photomicrograph of the control liver of adult albino rat showing normal portal triad consisting of a branch of portal vein (PV), branch of the hepatic artery (HA) and bile ductule (BD). (B) A photomicrograph of the liver of group II (CP) showing marked congestion of portal vein (blue arrow) with lymphocytic infiltration (black arrow). Hepatocytes necrotic changes such with marked hepatocytic pyknotic nuclei are also detected (red arrows) (C) A photomicrograph of group III (Green tea $+\mathrm{CP}$ ) showing the portal triads which almost similar to that of the control group. (Hx. \& E. (A), (B) \& (C) $\times 400$ ).

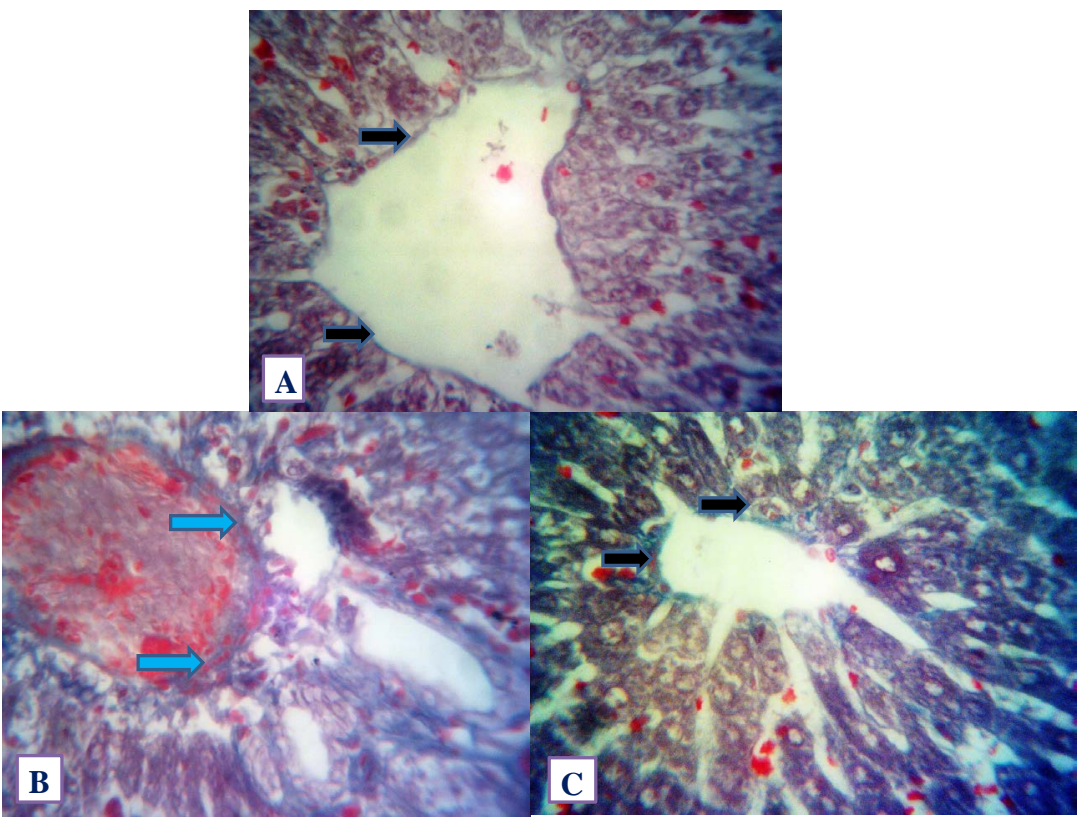

Figure 3. (A) A photomicrograph of the control liver of adult albino rat showing normal distribution of fine threads of collagen fibres around central vein (black arrows). (B) A photomicrograph of the liver of group II (CP) showing collagen fibres around central vein (blue arrows). (C) A photomicrograph of group III (Green tea + CP) showing distribution of fine threads of collagen fibres around central vein (black arrows). (Mallory's trichrome. (A), (B) \& (C) $\times 400)$. 


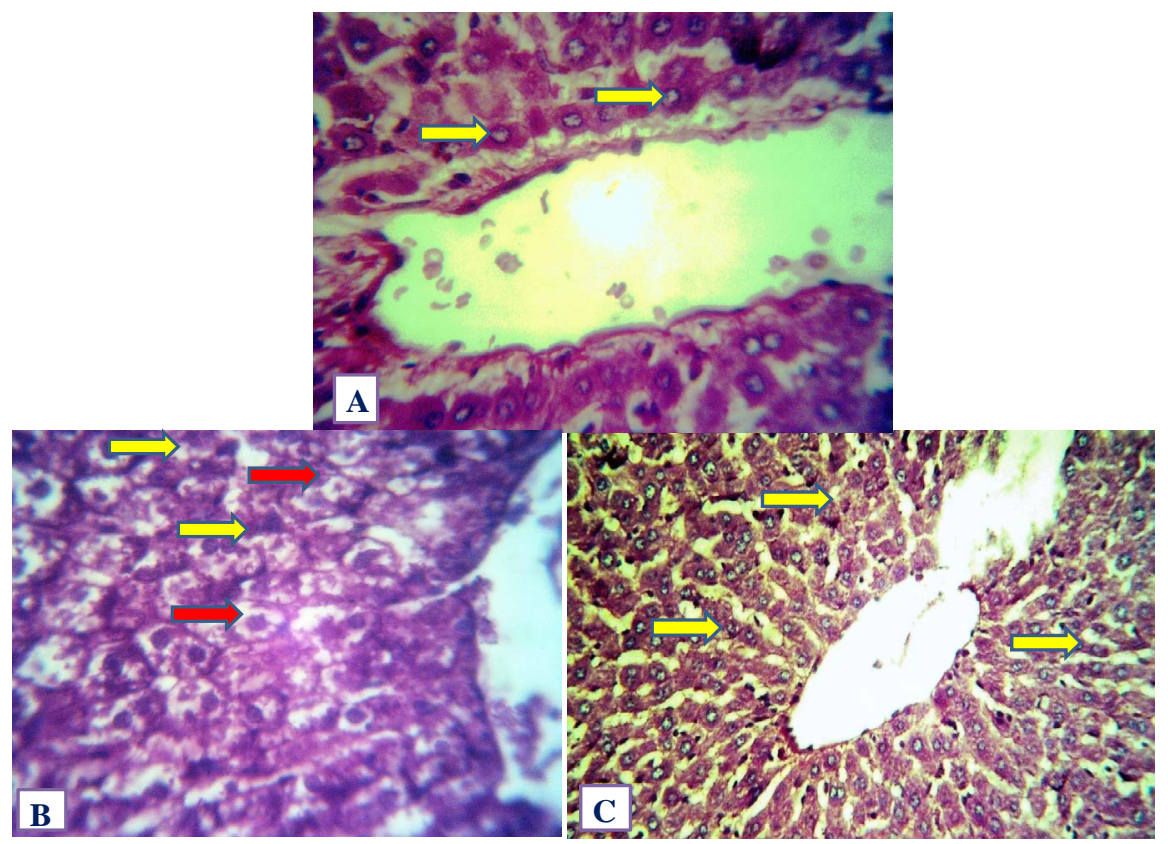

Figure 4. (A) A photomicrograph of the control liver of adult albino rat stained with Periodic Acid Schiff (PAS), showing strong PAS + ve granules in most of the hepatocytes. Carbohydrate granules appeared as coarse and fine pink granules in cytoplasm of hepatocytes (yellow arrows). (B) A photomicrograph of the liver of group II (CP) showing decreased amount of dispersed carbohydrates (red arrows). The amount of carbohydrates was mainly concentrated near the cell membrane of some hepatocytes (yellow arrows). (C) A photomicrograph of group III (Green tea + CP) showing reincrease in carbohydrates content of hepatocytes (yellow arrows). (PAS. (A) \& (B) $\times 400($ C) $\times 200$ ).

group (Figure 3(B)). In Group III the amount of collagenous fibres deposition seen around hepatic sinusoids, central vein and portal tract structures was significantly decreased in comparison to the normal group (Figure 3(C)).

Within Group I, investigations of normal liver sections stained with PAS revealed PAS + ve reaction (Figure 4(A)). However, carbohydrates were not uniformly distributed within the cytoplasm of these cells. Carbohydrates were found in the majority of the hepatocytes as coarse and fine pink granules. The nuclei showed negative affinity to the reaction (Figure 4(A)). In Group II, liver sections of rats showed that the amount of liver carbohydrates was significantly decreased in comparison to the control group (weak PAS + ve reaction) and carbohydrates were mainly concentrated near the cell membrane of some hepatocytes. Moreover, the most destructed hepatocytes showed weak reaction (Figure 4(B)). In Groups III, most of the hepatocytes revealed significant improvement of PAS + ve reaction in comparison to the cyclophosphamide group (Figure $4(C)$ ).

\section{Discussion}

$\mathrm{CP}$ is one of the commonly used anticancer drugs for its therapeutic efficacy against various cancers.

Our study revealed different degenerative hepatic structural changes in the 
group of rats treated by Cyclophosphamide (GII).

Our hepatic degenerative results seen in $\mathrm{CP}$ treated group might be caused by dilatation and congestion of the central hepatic and portal veins as well as the blood sinusoids of the liver. Vascular dilatation had been resulted from an increased prostaglandin formation with subsequent smooth muscle relaxation, whereas the vascular congestion might be resulted from a circulatory fluid loss followed by vascular engorgement with red cells [15].

Also, the present study displayed in CP treated group, a reduced hepatocytic glycogen content that might be pointed to the mitochondrial damage within the hepatocytes. Also, our study revealed a CP mediated excess of collagen fibrous formation, particularly in the portal tract area. Both observations (reduced glycogen and excess collagen) were consistent with the results of Sener et al [16] who stated that decreased glycogen content could be related to the liver damage induced by the released lysosomal hydrolytic enzymes from the hepatocytes following a stimulated lipid peroxidation. In addition, they mentioned that the lack of glycogen content might be caused by inhibiting the mitochondrial energy metabolism as well as hepatic inability either to store glycogen or to synthesize glycogen from lactate and pyruvate. They, as well, found that the vascular lesions which affected the hepatic perfusion could be a reason for hepatocytic degeneration and necrosis with subsequent reduction of total proteins and albumin formation causing their decreased concentrations in the serum.

Earlier studies have proved that ameliorative dose of CP could cause liver toxicity [17]. Experimental evidence recommends that oxidative stress is answerable for CP hepatotoxicity. It could generate reactive oxygen species (ROS) like superoxide anion, hydroxyl radical, and hydrogen peroxide $\left(\mathrm{H}_{2} \mathrm{O}_{2}\right)$ during its oxidative metabolism and discourages the antioxidant defense mechanism in liver [18]. A number of studies have shown that natural products with antioxidant activity protect against CP hepatotoxicity [19] [20] [21].

Regarding the green tea and CP treated group, liver sections were comparable to the control. Such improvement of the histological picture might be due to the antioxidant effect of green tea. This hypothesis is so far supported by the reports that green tea prevents hepatotoxicity [22]. The health-promoting effects of green tea are mainly attributed to its polyphenol content (catechin). Catechins reduced the formation of peroxides more effectively than alpha-tocopherol [23].

This hypothesis could be supported by Chacko et al. [23] who stated that green tea significantly decreases lipid peroxidation. Moreover, Shimizu et al. [24] reported that the anti-oxidative and anti-inflammatory activities are the key mechanisms of green tea catechins (polyphenols).

Finally, our present study observed, in group III markedly significant structural hepatic amelioration and protection compared with previously detected severe degenerative changes seen in group II. Furthermore, in that group there was an apparent improvement of liver architecture with some mild morphological changes like a mild dilatation of portal vein, a mild periportal mononuclear cellular infiltration, and a very few perivenous and portal collagen fibers formation. 


\section{Conclusions}

The present study identified pathological features in the rat liver treated with CP. It also provided an evidence of the possible hepatoprotective effect of green tea against the hepatotoxic effect of CP. Therefore, supplementation of green tea to $\mathrm{CP}$ treated patients should be routinely advised in order to protect and to cure their livers against such undesirable effects of that medication. Further study is necessary to elucidate target compounds from green tea responsible for liver protection.

In addition, human investigations are recommended to be carried out to demonstrate whether similar results could be obtained.

\section{References}

[1] Hamden, K., Carreau, S., Ellouz, F., et al. (2009) Improvement Effect of Green Tea on Hepaticdysfunction, Lipid Peroxidation and Antioxidant Defence Depletion Induced by Cadmium. African Journal of Biotechnology, 8, 4233-4238.

[2] Elzoghby, R.R., Ahlam, F.H., Abdel-Fatah, A., et al. (2014) Protective Role of Vitamin $\mathrm{C}$ and Green Tea Extract on Malathion-Induced Hepatotoxicity and Nephrotoxicity in Rats. American Journal of Pharmacology and Toxicology, 9, 177-188. https://doi.org/10.3844/ajptsp.2014.177.188

[3] Adel, A.G., Sabah, E.F., Tarek, S. and El-Naggar, A. (2009) Protective Role of Green Tea on Carbon Tetrachloride-Induced Acute Hepatotoxicity. Scientific Journal of Faculty of Science (Minufiya University), 23, 55-67.

[4] Higdon, J.V. and Frei, B. (2003) Tea Catechins and Polyphenols: Health Effects, Metabolism, and Andantioxidant Functions. Critical Reviews in Food Science and Nutrition, 43, 89-143.

[5] Heikal, T.M., Mossa, A.-T.H., Rasoul, M.A.A., et al. (2013) The Ameliorating Effects of Green Tea Extract against Cyromazine and Chlorpyrifos Induced Liver Toxicity in Malerats. Changes, 5, 9.

[6] Al-Attar, A.M. and Abu Zeid, I.M. (2013) Effect of Tea (Camellia sinensis) and Olive (Oleaeuropaea L.) Leaves Extracts on Male Mice Exposed to Diazinon. BioMed Research International, 461, 415-420.

[7] Kuriyama, S., Shimazu, T., Ohmori, K., et al. (2006) Green Tea Consumption and Mortality Due to Cardiovascular Disease, Cancer, and All Causes in Japan: The Ohsaki Study. JAMA, 296, 1255-1265.

[8] Frei, B. and Higdon, J.V. (2003) Antioxidant Activity of Tea Polyphenols in Vivo: Evidence from Animal Studies. The Journal of Nutrition, 133, 3275S-3284S. https://doi.org/10.1093/jn/133.10.3275S

[9] Lotito, S.B. and Frei, B. (2006) Consumption of Flavonoid-Rich Foods and Increased Plasma Antioxidant Capacity in Humans: Cause, Consequence, or Epiphenomenon? Free Radical Biology and Medicine, 41, 1727-1746. https://doi.org/10.1016/j.freeradbiomed.2006.04.033

[10] Ilbey, Y.O., Ozbek, E., Simsek, A., Otunctemur, A., Cekmen, M. and Somay, A. (2009) Potential Chemoprotective Effect of Melatonin in Cyclophosphamide- and Cisplatin-Induced Testicular Damage in Rats. Fertility and Sterility, 92, 1124-1132. https://doi.org/10.1016/j.fertnstert.2008.07.1758

[11] Kirkland, R.T., Bongiovanni, A.M., Cornfeld, D., McCormick, J.B., Parks, J.S. and 
Tenore, A. (1976) Gonadotropin Responses to Luteinizing Releasing Factor in Boys Treated with Cyclophosphamide for Nephrotic Syndrome. The Journal of Pediatrics, 89, 941-944. https://doi.org/10.1016/S0022-3476(76)80600-2

[12] Kaur, R., Sangha, G. and Bilaspuri, G. (1997) Cyclophosphamide Induced Structural and Biochemical Changes in Testis and Epididymidis of Rats. IJEB, 35, 771-775.

[13] Masala, A., Faedda, R., Alagna, S., Satta, A., Chiarelli, G., Rovasio, P.P., Ivaldi, R., Taras, M.S., Lai, E. and Bartoli, E. (1997) Use of Testosterone to Prevent Cyclophosphamide-Induced Azoospermia. Annals of Internal Medicine, 126, 292-295. https://doi.org/10.7326/0003-4819-126-4-199702150-00005

[14] Bancroft, D. and Gamble, M. (2008) Bancroft's Theory and Practice of Histological Techniques. 6th Edition, Elsevier, Churchill Livingstone, Edinburgh, Scotland, 178-186, 221-224.

[15] Walter, T. and Talbot, I. (1996) Welter and Israel General Pathology. Churchill Livingstone, London, 167.

[16] Şener, G., Ekşioğlu-Demiralp, E., Çetiner, M., et al. (2006) $\beta$-Glucan Ameliorates Methotrexate Induced Oxidative Organ Injury via Its Antioxidant and Immunomodulatory Effects. European Journal of Pharmacology, 542, 170-178.

https://doi.org/10.1016/j.ejphar.2006.02.056

[17] Mahsa, Z. and Shivanandappa, T. (2013) Amelioration of CyclophosphamideInduced Hepatotoxicity by the Root Extract of Decalepis hamiltonii in Mice. Food and Chemical Toxicology, 57, 179-184. https://doi.org/10.1016/j.fct.2013.03.028

[18] Bhattacharya, A., Lawrence, R.A., Krishnan, A., Zaman, K., Sun, D. and Fernandes, G. (2003) Effect of Dietary n-3 and n-6 Oils with and without Food Restriction on Activity of Antioxidant Enzymes and Lipid Peroxidation in Livers of Cyclophosphamide Treated Autoimmune-Prone NZB/W Female Mice. Journal of the American College of Nutrition, 22, 388-399. https://doi.org/10.1080/07315724.2003.10719322

[19] Haque, R., Bin-Hafeez, B., Parvez, S., Pandey, S., Sayeed, I., Raisuddin, S. and Raisuddin, S. (2003) Aqueous Extract of Walnut (Juglans regia L.) Protects Mice against Cyclophosphamide Induced Biochemical Toxicity. Human \& Experimental Toxicology, 22, 473-480. https://doi.org/10.1191/0960327103ht388oa

[20] Kumar, K.B.H. and Kuttan, R. (2005) Chemoprotective Activity of an Extract of Phyllanthus amarus against Cyclophosphamide Induced Toxicity in Mice. Phytomedicine, 12, 494-500. https://doi.org/10.1016/j.phymed.2004.03.009

[21] Sharma, N., Trikha, P., Athar, M. and Raisuddin, S. (2000) Inhibitory Effect of Emblica officinalis on the in Vivo Clastogenicity of Benzo[a]pyrene and Cyclophosphamide in Mice. Human \& Experimental Toxicology, 19, 377-384.

https://doi.org/10.1191/096032700678815945

[22] Crespy, V. and Williamson, G. (2004) A Review of the Health Effects of Green Tea Catechins in in Vivo Animal Models. The Journal of Nutrition, 134, 3431S-3440S. https://doi.org/10.1093/jn/134.12.3431S

[23] Chacko, S.M., Thambi, P.T., Kuttan, R. and Nishigaki, I. (2010) Beneficial Effects of Green Tea: A Literature Review. Chinese Medicine, 5, 13.

https://doi.org/10.1186/1749-8546-5-13

[24] Shimizu, M., Shirakami, Y., Sakai, H., Kubota, M., Kochi, T., et al. (2015) Chemopreventive Potential of Green Tea Catechins in Hepatocellular Carcinoma. International Journal of Molecular Sciences, 16, 6124-6139. https://doi.org/10.3390/ijms16036124 